\title{
Guanylate cyclase 2C agonism corrects CFTR mutants
}

\author{
Kavisha Arora, ${ }^{1}$ Yunjie Huang, ${ }^{1}$ Kyushik Mun, ${ }^{1}$ Sunitha Yarlagadda, ${ }^{1}$ Nambirajan Sundaram, ${ }^{2}$ \\ Marco M. Kessler, ${ }^{3}$ Gerhard Hannig, ${ }^{3}$ Caroline B. Kurtz, ${ }^{4}$ Inmaculada Silos-Santiago, ${ }^{5}$ \\ Michael Helmrath, ${ }^{2}$ Joseph J. Palermo, ${ }^{6}$ John P. Clancy, ${ }^{1}$ Kris A. Steinbrecher, ${ }^{7}$ \\ and Anjaparavanda P. Naren ${ }^{1}$ \\ 'Division of Pulmonary Medicine, Department of Pediatrics, and 2Department of Pediatric General and Thoracic Surgery, \\ Cincinnati Children's Hospital Medical Center (CCHMC), Cincinnati, Ohio, USA. Ironwood Pharmaceuticals, Cambridge, \\ Massachusetts, USA. ${ }^{4}$ Synlogic, Cambridge, Massachusetts, USA. ${ }^{5}$ Decibel Therapeutics, Boston, Massachusetts, USA \\ ${ }^{6}$ Division of Gastroenterology, Hepatology and Nutrition, Department of Pediatrics, and 'Division of Gastroenterology, \\ Hepatology and Nutrition, CCHMC, Cincinnati, Ohio, USA.
}

Cystic fibrosis (CF) is a genetic disorder in which epithelium-generated fluid flow from the lung, intestine, and pancreas is impaired due to mutations disrupting CF transmembrane conductance regulator (CFTR) channel function. CF manifestations of the pancreas and lung are present in the vast majority of CF patients, and $15 \%$ of CF infants are born with obstructed gut or meconium ileus. However, constipation is a significantly underreported outcome of CF disease, affecting $47 \%$ of the CF patients, and management becomes critical in the wake of increasing life span of CF patients. In this study, we unraveled a potentially novel molecular role of a membrane-bound cyclic guanosine monophosphate-synthesizing (cCMP-synthesizing) intestinal enzyme, guanylate cyclase 2 C (CCC) that could be targeted to ameliorate CF-associated intestinal fluid deficit. We demonstrated that CCC agonism results in functional rescue of murine F508del/F508del and R117H/R117H Cftr and CFTR mutants in CF patient-derived intestinal spheres. CCC coexpression and activation facilitated processing and ER exit of F508del CFTR and presented a potentially novel rescue modality in the intestine, similar to the CF corrector VX-809. Our findings identify CCC as a biological CFTR corrector and potentiator in the intestine.

Conflict of interest: APN received grant support from Ironwood Pharmaceuticals.

Submitted: March 3, 2017

Accepted: August 29, 2017

Published: October 5, 2017

\section{Reference information:}

JCI Insight. 2017;2(19):e93686

https://doi.org/10.1172/jici.

insight.93686.

\section{Introduction}

Cystic fibrosis (CF) affects individuals with an inherent loss of chloride/bicarbonate transport function of the CF transmembrane conductance regulator (CFTR), resulting in a general manifestation of mucus buildup on the epithelial surfaces of the lung, pancreas, and intestine $(1,2)$. CF mortalities usually coincide with the collapse of lung function. Nevertheless, gastrointestinal-related (GI-related) issues may persist throughout the course of CF. Meconium ileus (MI), constipation, distal intestinal obstruction syndrome (DIOS), and gastroesophageal reflux disease (GERD) are the usual GI problems observed in CF (3). Sadly, CF-associated constipation is recognized much less than is needed, is reported to affect $47 \%$ of the CF population, and demands timely management to ensure overall well-being of CF patients (4). Hence, it is important to study the mechanisms that benefit GI transit in CF and worth identifying pathways that can be specifically targeted in the GI system without a multisystemic interference in CF. Guanylate cyclase 2C (GCC), the receptor for the endogenous hormones guanylin and uroguanylin and heat-stable enterotoxins from E. coli (STa) is predominantly expressed in intestinal epithelial cells, with no expression reported in other epithelial cell types (5). Activation of GCC results in elevated levels of intracellular cyclic guanosine monophosphate (cGMP), which can stimulate CFTR-mediated chloride ion transport into the intestinal lumen to drive water transit (6-9). GCC mutations are linked to obstructed bowel or MI and diarrhea in similar populations $(10,11)$. Also, the secretory impetus of GCC agonist (linaclotide, Lc) in the intestine is clinically exploited to alleviate constipation in irritable bowel syndrome (IBS) patients $(12,13)$.

F508del, the most common CF-causing mutation in CFTR, generates a pronounced processing defect and ER retention, transitioning it to a proteosomal degradation pathway (14). VX-809 (Lumacaftor), termed a CF corrector, improves folding and surface levels of F508del CFTR $(15,16)$. Other 
prevalent CF mutations, which result in obliterated (G551D CFTR) or reduced (R117H CFTR) channel function at the cell surface, are responsive to CF potentiators such as VX-770 (ivacaftor) $(17,18)$. Irrespective of their fundamental defects, both F508del CFTR and R117H CFTR mutant proteins would ideally derive more clinical benefit from the combining effects of a corrector and increased cell surfaceassociated function $(15,17-19)$.

Using ex vivo (organoid culture) and in vivo (ileal closed loop) models to study functional mechanisms of the intestine, we unraveled a potentially novel molecular role of GCC in promoting rescue of F508del CFTR from ER retention. This pathway may further imply that the predominant expression of GCC and its activation in the gut would help alleviate $\mathrm{CF}$-associated constipation and, overall, would be therapeutic, specifically to the GI system in CF.

\section{Results}

GCC activation stimulates CFTR function in intestinal stem cell model. Using RNA sequencing, we determined the expression levels of various guanylate cyclases and identified GCC as the most predominant member present in the ileum, while not significantly present in the large airways (trachea) of mice (Figure 1, A and B). This finding suggests that GCC signaling may have a specialized role in regulating intestinal physiology. To study this aspect of functional relevance of GCC in the gut, we synthesized a 14-amino acid GCC agonist peptide STcore (STc), which contains the GCC binding/activating region of heat-stable enterotoxin 2 (STa2) and a peptide very similar to clinically used Lc (Supplemental Figure 1A; supplemental material available online with this article; https://doi.org/10.1172/jci.insight.93686DS1). STc- and Lc-activated GCC overexpressed recombinantly in HEK 293 cells and generated cGMP production of a similar caliber, but not in HEK 293 control cells (Supplemental Figure 1, B and C), confirming GCC-specific action of these peptide analogs. To establish the significance of the GCC pathway in intestinal signaling, we used an intestinal organoid culture method. Intestinal organoid culture is an elegant method to grow intestinal crypts in a laminin/collagen-rich matrix, which allow the crypts to form an enclosed structure called an enterosphere with a central lumen surrounded by the cell types of the mature intestinal epithelium (20, 21). Importantly, these structures represent key features of the in vivo structure and function of the source tissue. This closed structure of an enterosphere encompassing a central lumen allows investigators to study fluid dynamics (i.e., secretion and absorption) caused by physiological alterations in the epithelia that can result in either swelling or shrinkage of the enterospheres (22). It turned out that enterospheres are extremely useful models to study CFTR function in which forskolin, a cAMP agonist, induces rapid swelling of the enteropsheres, with normal CFTR caused by fluid filling the central lumen as CFTR becomes active (23-25). Loss-of-function CFTR mutants fail to stimulate secretion in such an assay as demonstrated by us and others $(23,24)$. We studied the effect of GCC activation on CFTR-dependent fluid secretion and found that incubation with STc stimulated swelling in the WT/WT Cftr enterospheres (Figure 1, C and D). To confirm that STc-stimulated fluid secretion depends on GCC activation, we isolated enterospheres

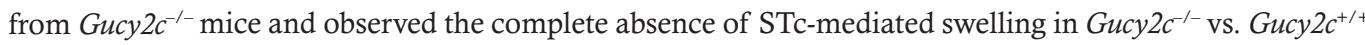
spheres without any apparent loss of stimulated fluid secretion in response to cell-permeant stimulants 8-Br-cGMP and cAMP agonist forskolin (Figure 1, C and D). Next, we demonstrated GCC localization primarily on the inward-facing apical surface of the enterosphere, with markedly less expression detected on the basolateral side (Figure 1E). This differential GCC expression pattern led us to investigate the potential differences in GCC-stimulated secretion following the addition of GCC agonist peptide either to the basolateral or apical side of an enterosphere. Apical administration of STc by microinjection into the lumen of the enterosphere stimulated secretion at a level comparable with basolateral administration (Figure $1, \mathrm{~F}$ and $\mathrm{G})$. Using a biotinylated peptide analog of STc, biotin-Lc, we found that biotin-Lc could transcytose across the epithelial cell lining of the enterosphere (Supplemental Figure 2, A and B). This apparent transcytosis pathway may explain the lack of difference in fluid secretion, irrespective of the side of ligand application to the enterosphere (Supplemental Figure 2, A and B). Next, we tested whether STc-mediated intestinal swelling is caused by CFTR activation. STc-dependent secretion decreased in the presence of the CFTR inhibitor CFTRinh-172 (Figure 1H). CF mice (F508del/F508del Cftr) do not have CF-associated lung pathology but are valuable for studying CF-related intestinal obstruction, and there is a high incidence of postweaning mortality associated with MI ( $60 \%$ occurrence rate) (4). Treatment with STc failed to stimulate secretion in the enterospheres isolated from F508del/F508del Cftr mice and stimulated secretion only following incubation of F508del/F508del enterospheres at low temperature $\left(28^{\circ} \mathrm{C}, 24\right.$ hours), which 

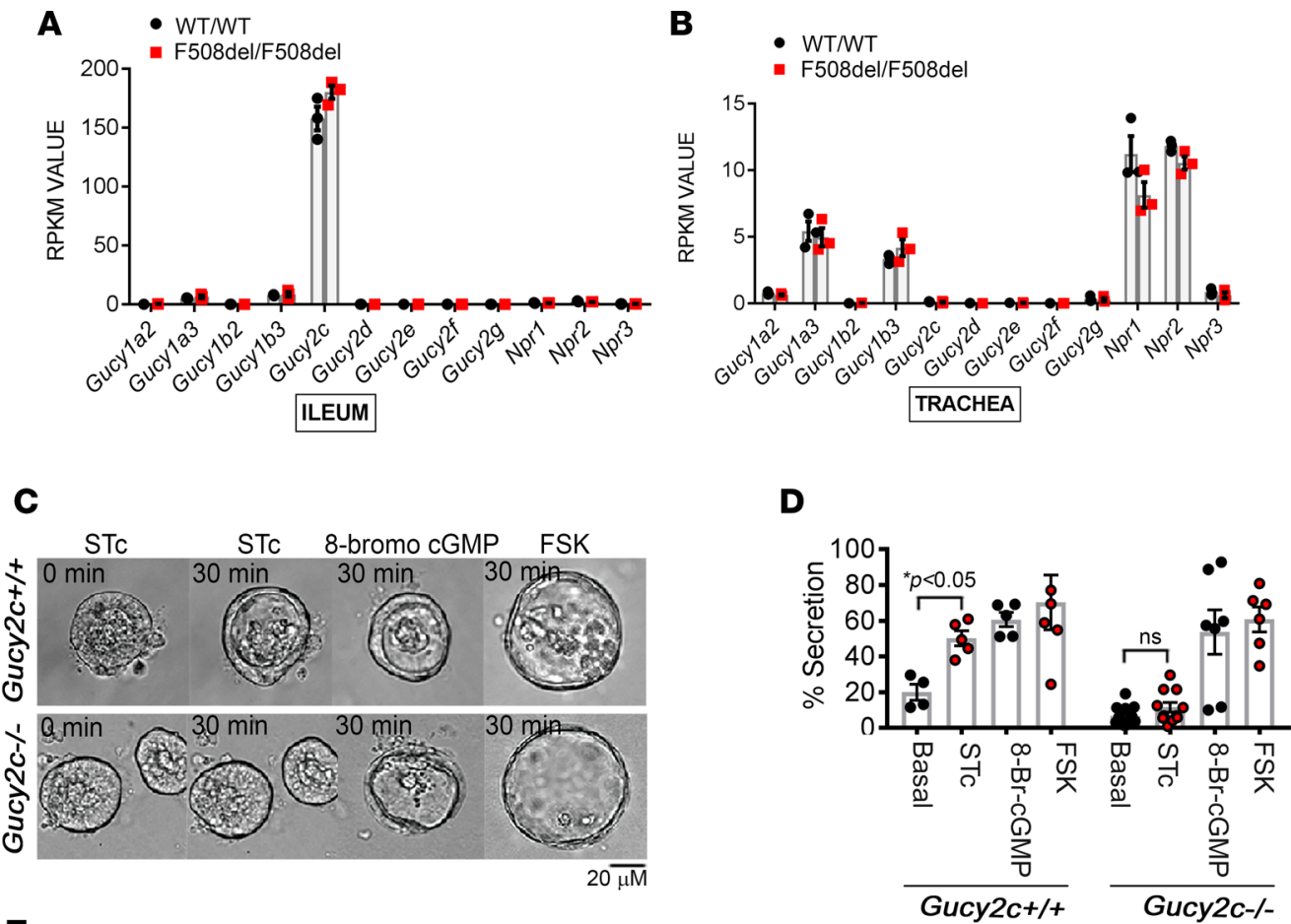

E Mouse intestinal organoid
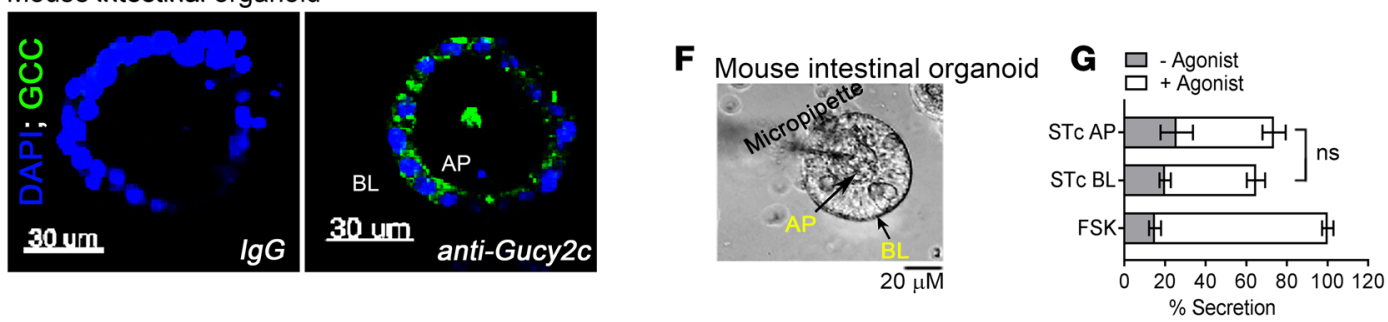

H High-content microscopy
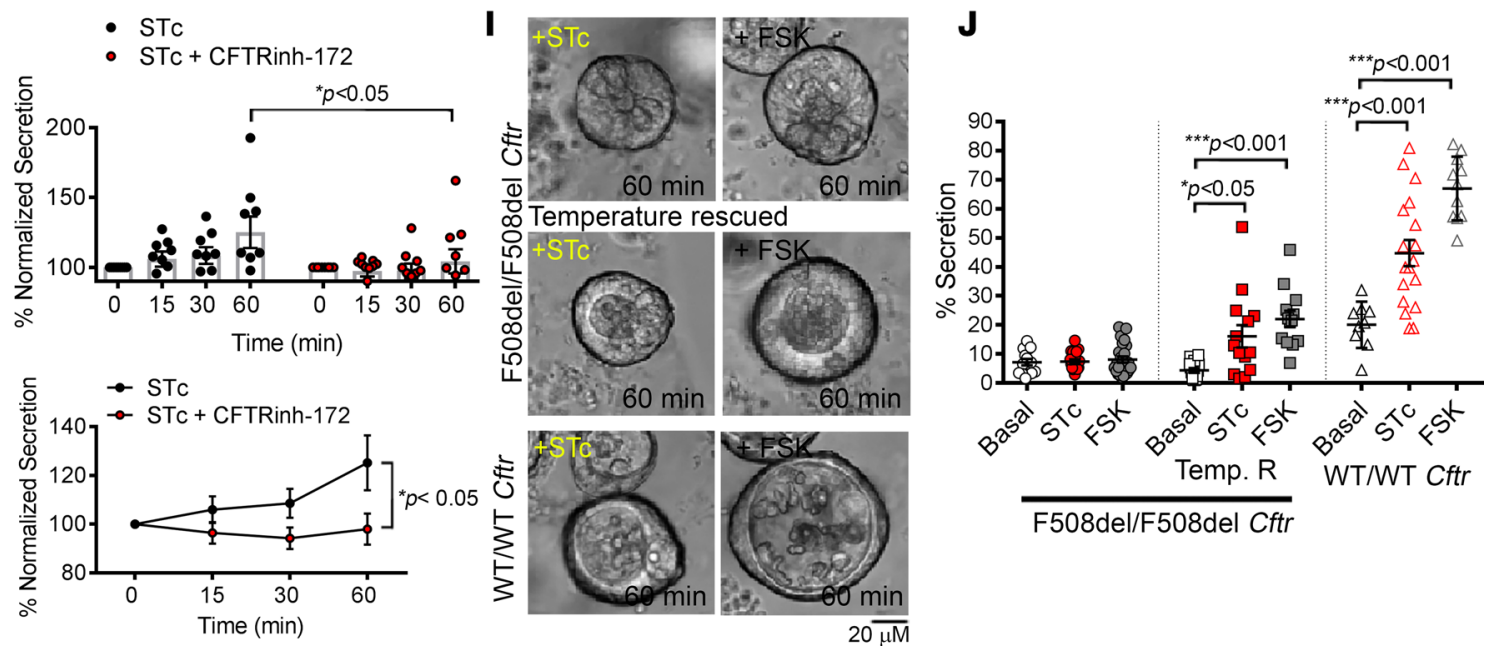

Figure 1. Validation of intestinal spheres as model to study GCC-stimulated fluid secretion. RNA-seq analysis demonstrates relative abundance of transcripts of various guanylate cyclases in (A) ileum and (B) trachea isolated from WT/WT Cftr $(n=3)$ and F508del/F508del Cftr ( $n=3)$ mice.

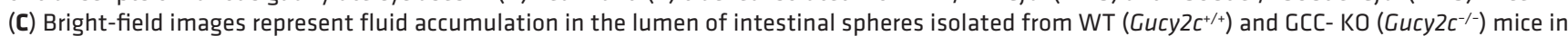
response to various CFTR activating agonists. (D) Bar graph shows quantitation of fluid secretion in intestinal spheres corresponding to basal or no stimulation and in response to STc $(500 \mathrm{nM}, 30 \mathrm{~min}), 8-\mathrm{Br}-\mathrm{CGMP}(250 \mu \mathrm{M}, 30 \mathrm{~min})$, and forskolin (FSK, $10 \mu \mathrm{M}, 30 \mathrm{~min})$, all added in the media and calculated from $n=5-10$ enterospheres from 2 mice in each group. Data is \pm SEM with $P$ value by 1-way ANOVA with Bonferroni's adjustment for multiple comparisons. (E) Confocal images of intestinal spheres showing GCC-specific immunostaining predominantly present on the apical side of the spheres. (F) Image of an intestinal sphere depicting microinjection of STc into the lumen of the structure. BL, basolateral; AP, apical. (G) Bar graph shows quantitation of fluid secretion in intestinal spheres corresponding to basal or no stimulation and in response to STc delivered in the media or microinjected into the lumen calculated from $n=5$ enterospheres. Response to FSK was tested after adding the reagent into the media. Data is \pm SEM with $P$ value 
by 1-way ANOVA with Bonferroni's adjustment for multiple comparisons. (H) Dot blot and line-graph depict fluid secretion calculated as the total area of intestinal spheres ( $n=9-11$ enterospheres) following activation of CFTR function by using STc (500 nM, 30 min) in the presence and absence of CFTRinh-172 (20 $\mu \mathrm{M}, 30 \mathrm{~min})$ in an assay done in a high-content microscope. Data is \pm SEM with $P$ value by 2 -tailed unpaired Student's $t$ test. (I) FSK- and STc-dependent swelling of intestinal spheres isolated from CFTR-mutant mice F508del/F508del Cftr was tested. A set of F508del/F508del Cftr intestinal spheres was subjected to low-temperature incubation ( $28^{\circ} \mathrm{C}, 24 \mathrm{~h}$ ) and subsequently tested for FSK- (10 $\mu \mathrm{M}$, 30 min) and STc-dependent (500 $\mathrm{nM}, 30 \mathrm{~min}$ ) secretion. (J) Dot plot represents quantitation of the fluid secretion in $n=9-17$ enterospheres per condition described in $\mathbf{I}$. Temp. R, temperature rescue. Data is \pm SEM with $P$ value determined using 1-way ANOVA with Bonferroni's test for multiple comparisons.

permits an escape of ER-retained F508del CFTR protein (ref. 26 and Figure 1, I and J). Both STc and Lc stimulated the production of cGMP in WT/WT Cftr and F508del/F508del Cftr enterospheres to a similar extent, suggesting no apparent difference in GCC activation potential between these 2 genetic backgrounds (Supplemental Figure 2C). Transcript levels of GCC did not differ between WT/WT Cftr and F508del/ F508del Cftr enterospheres (Supplemental Figure 2D). Hence, STc/Lc-mediated secretion in enterospheres is GCC driven and CFTR dependent.

Long-term GCC agonist exposure corrects CFTR mutant proteins. Given the fact that Lc consumption over a 26-week treatment span could significantly accelerate fluid transit in IBS patients (12), we investigated whether long-term exposure to STc could have an impact on mutant CFTR function. We first studied the effect of STc on F508del CFTR protein quality and activity. We found that recombinant expression of GCC in HEK 293 cells stably expressing F508del CFTR increased ER form band B as well as mature band C of F508del CFTR and, consistent with an enhanced membrane-localized population of F508del CFTR, exhibited an effect similar to that of VX-809 (Figure 2, A and B). Incubation of F508del CFTR HEK 293 cells with STc (500 nM, 24 hours) enhanced the rescue of F508del CFTR protein only in GCC-expressing cells and upon a low temperature shift (Figure 2A). GCC coexpression and activation enhanced chloride transport activity of F508del CFTR measured using (6-methoxy-N-[3-sulfopropyl' quinolinium; SPQ) chloride indicator (Figure 2C). An extensive colocalization was observed between F508del CFTR and GCC, especially in the post-ER compartments (Supplemental Figure 2E). Based on our quantum dot-based (Q dot-based) tracking of biotin-Lc, we observed internalization and accumulation of $\mathrm{Q}$ dot-labeled biotin-Lc in ER/post-ER compartments and colocalization with F508del CFTR protein clusters (Supplemental Figure 2 F). Notably, GCC coexpression also enhanced the protein expression of WT CFTR, suggesting that the correcting potential of GCC is not dependent on the presence of a folding defect mutation like F508del in CFTR (Supplemental Figure 3A). To establish the functional relevance of GCC-mediated correction of F508del CFTR, mouse F508del/F508del Cftr enterospheres were treated with increasing concentrations of STc (50-1,000 nM) for 24 hours (Figure 3, A and B). We observed a concentration-dependent increase in fluid secretion in F508del/F508del Cftr enterospheres upon short-term stimulation with forskolin (10 $\mu \mathrm{M}, 30 \mathrm{~min}$ ) (Figure 3, A and B). The rescued enterosphere-swelling response was abrogated at a STc dose $<500 \mathrm{nM}$ and mitigated at a dose of 1,000 nM in the F508del/F508del Cftr enterospheres treated with CFTRinh-172 prior to stimulation with forskolin (Figure 3B). Based on this observation, there may be a component of CFTR-independent rescue of secretion by STc at a concentration of 1,000 $\mathrm{nM}$ in the intestinal organoids, most likely through regulation of $\mathrm{Na}^{+}$absorption. Treatment of F508del/F508del Cftr enterospheres with STc (500 nM, 24 hours) did not significantly alter CFTR, GCC, NHE3, and Lgr5 (intestinal stem cell marker) transcripts (Supplemental Figure 3B). Using CFTR-specific immunostaining, we found that STc treatment partially resolved F508del CFTR protein aggregates in F508del/F508del CFTR enterospheres and improved membrane localization of the mutant protein (Figure 3C). Next, we tested whether GCC activation-mediated functional correction is selective to the F508del CFTR mutant protein because of its inherent folding problem. Following a 24-hour prior incubation period with STc (50 nM), an increased CFTR-dependent fluid secretion was also observed in mouse R117H/R117H Cftr enterospheres (Figure 3, D and E). This finding may be relevant to our previous observation that GCC also increases the protein levels of fold-normal CFTR (Supplemental Figure 3A). Together, these observations provide important insights into the effects of GCC agonism in rescuing CFTR mutants independent of the type of mutation - findings not previously linked to this mechanism, which may support a novel, generic outcome of GCC agonism on CFTR-dependent intestinal physiology.

Using a closed intestinal loop model, we further demonstrated the in vivo relevance of Lc administration in the functional restoration of F508del CFTR. Cholera toxin (CTX), an enterotoxin from vibrio cholerae that can cause excessive CFTR functional activation via stimulation of cAMP production, 
A

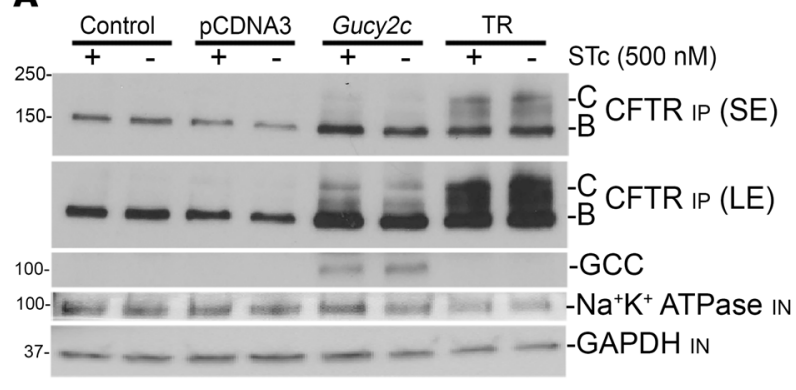

C

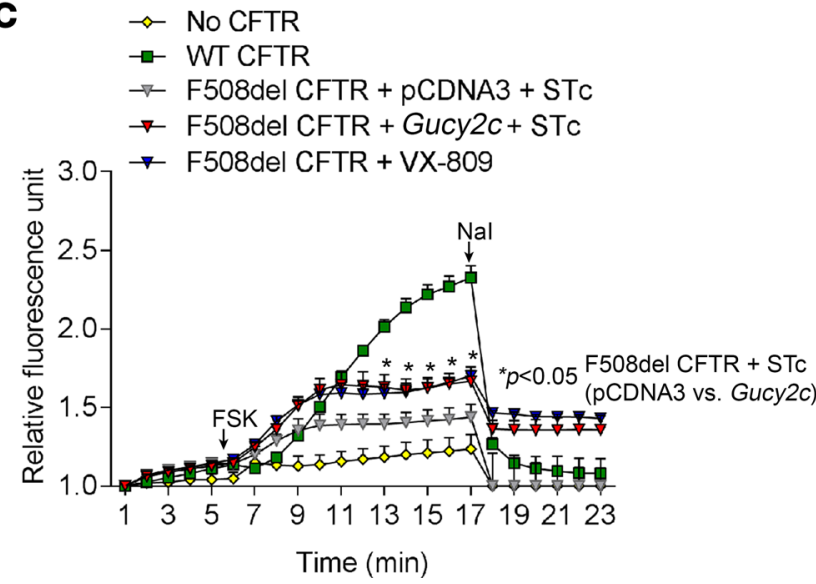

B
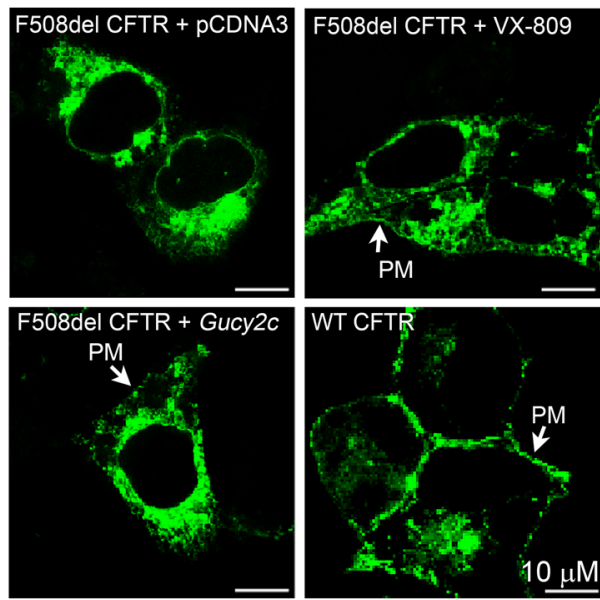

Figure 2. Long-term exposure to STc corrects and rescues F508del CFTR mutants. (A) Western blot data to study the amount of FLAC F508del CFTR B and C with and without recombinant expression of GCC or treatment with GCC agonist STc ( $500 \mathrm{nM}, 24 \mathrm{~h}$ ) in HEK 293 cells. A set of F508del CFTR-expressing cells was subjected to temperature rescue (TR). CFTR was immunoprecipitated using resin-conjugated with 24-1 anti-CFTR antibody and probed using anti-FLAG $M_{2}$-HRP. This experiment was independently repeated 5 times and generated similar result. SE, short-exposure; LE, long exposure; IN, input. (B) Representative confocal images of YFP-tagged F508del CFTR expressed in HEK 293 cells under the following conditions: empty vector (pCDNA3), HA-CCC coexpression, and VX-809 treatment $(2 \mu \mathrm{M}, 24 \mathrm{~h})$. YFP WT CFTR was expressed in the cells separately to mark intensive localization of a fold-normal CFTR protein at the plasma membrane (PM, indicated by white arrows). (C) Dequenching of SPQ fluorescence to study chloride transport activity of CFTR in HEK 293 cells upon stimulation with forskolin $(10 \mu \mathrm{M})$ expressing no CFTR, WT-CFTR, F508del CFTR + pCDNA3 treated with STc (500 nM, 24 h), F508del CFTR + GCC treated with STc $(500 \mathrm{nM}, 24 \mathrm{~h})$, and F508del CFTR treated with VX-809 $(2 \mu \mathrm{M}, 24 \mathrm{~h})$. SPQ signal was quenched using Nal at the end of the experiment. Error bars represent \pm SEM calculated from $n=6$ wells from 3 independent experiments. $P$ value by 1-way ANOVA with Bonferroni's adjustment for multiple groups.

when injected into a closed loop will cause release of fluid in the loop that can be weighed to assess CFTR function (9). F508del/F508del Cftr mice received an oral gavage containing either PBS or Lc $(150 \mu \mathrm{g} / \mathrm{kg})$ and were subjected to CTX-loop experiments and short-circuit (Isc) measurements after 24 hours; WT/WT Cftr mice served as controls (Figure 4A). Lc-treated mice accumulated 3.6-fold more fluid in the loop, compared with almost no secretion in the PBS-treated group (Figure 4, B and C). Correction of F508del CFTR is typically assessed by the restored amounts of cAMP-stimulated $\mathrm{Cl}^{-}$ currents. CFTR-dependent $\mathrm{Cl}^{-}$currents were monitored in jejunal and ileal segments separated from PBS- and Lc-oral gavaged (PBS-OG and Lc-OG) F508del/F508del Cftr mice and control WT/WT Cftr mice (Figure 4, D and E). CFTR-dependent $\mathrm{Cl}^{-}$currents were restored by 4.2 -fold in Lc-subjected compared with PBS-subjected intestinal segments (Figure 4E). Hence, Lc partially restores mutant CFTR function in the gut.

Long-term GCC agonist exposure rescues mutant CFTR function in CF patient-derived intestinal organoids. Patient-derived duodenal biopsies are a valuable resource for initiating long-term intestinal culture, and the generated organoids/enterospheres closely mimic the functional profile of the donor intestine. To establish the therapeutic relevance of GCC activation to $\mathrm{CF}$, we isolated human intestinal spheres from patient biopsies carrying the F508del/F508del CFTR mutation (patient 1), F508del/R117H 7T/9T CFTR mutation (patient 2), and G542X/R74W, V201M, D1270N mutation, an example of a complex 
A $(+)$ FSK STc, 24 h

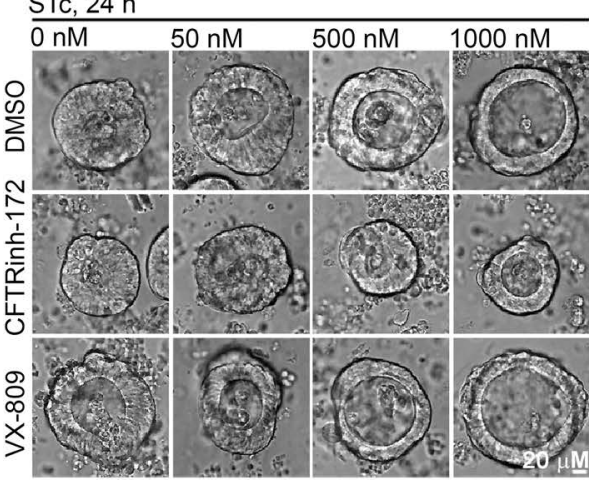

C

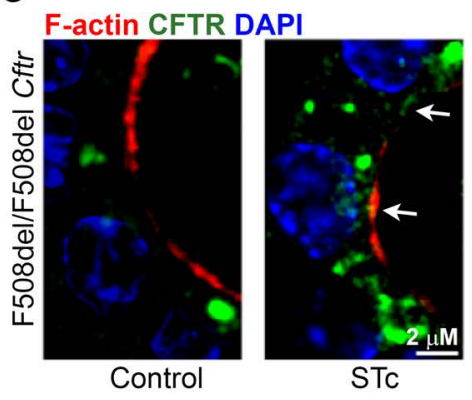

D

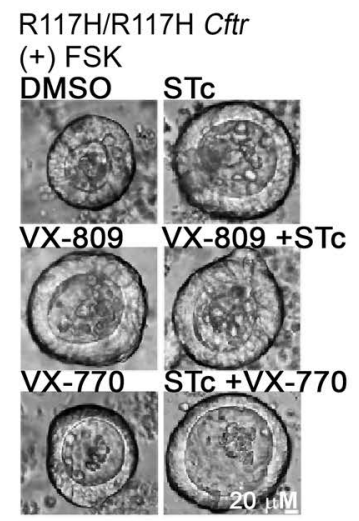

B

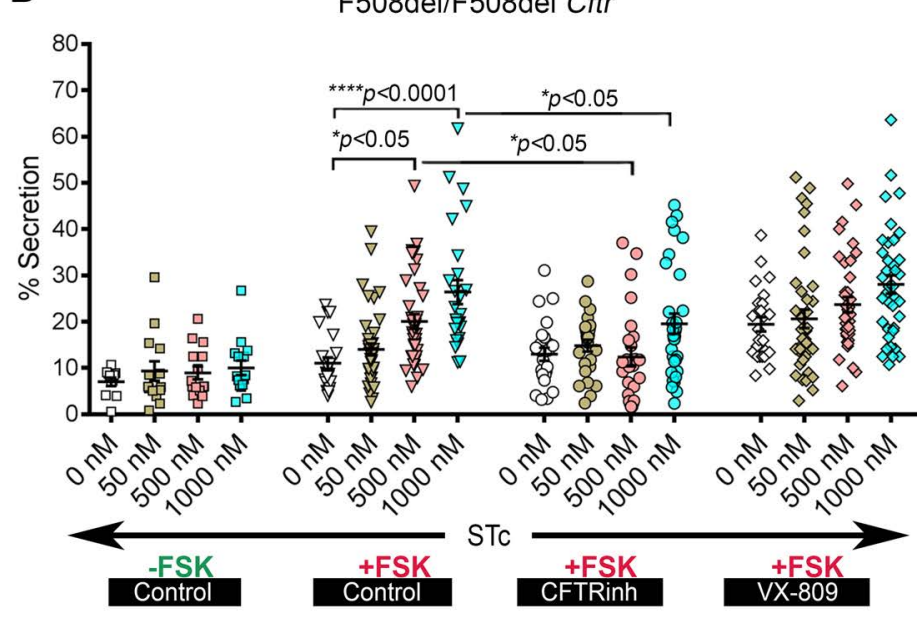

$(+)$ FSK

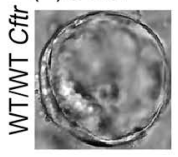

F508del/F508del Cftr

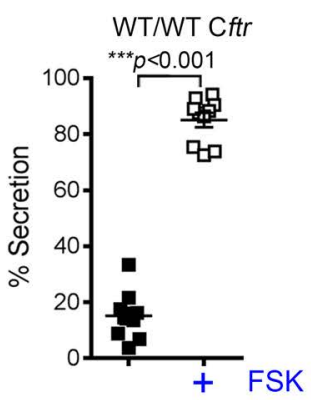

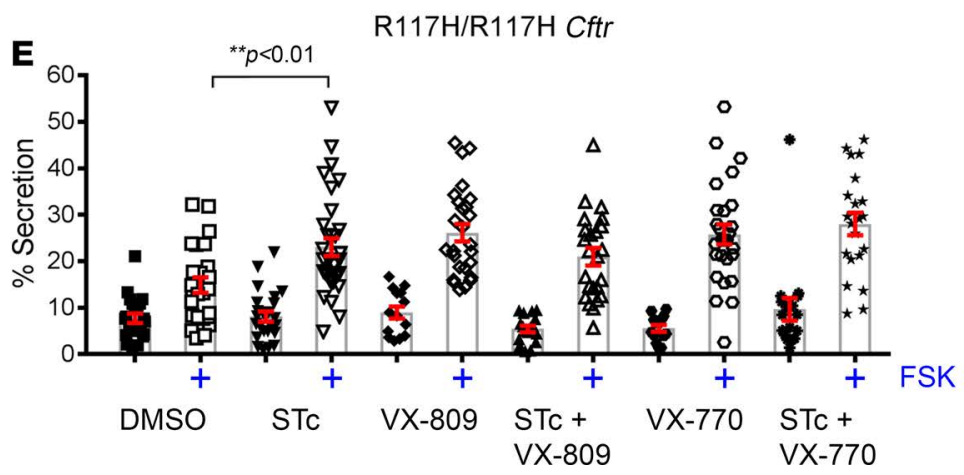

Figure 3. Long-term exposure to STc corrects and rescues F508del CFTR function in enterospheres. (A) Representative images of F508del/F508del Cftr enterospheres depict secretion under various treatment conditions: (top row) STc (0,50,500, and 1,000 nM, $24 \mathrm{~h})$, (middle) STc (0, 50, 500, and 1,000 $\mathrm{nM}, 24 \mathrm{~h})+$ CFTRinh-172 (20 $\mu \mathrm{M}, 30 \mathrm{~min})$, and (bottom) STc (0, 50, 500, and 1,000 nM, $24 \mathrm{~h})+\mathrm{VX}-809(2 \mu \mathrm{M}, 24 \mathrm{~h})$. (B) Dot plot represents quantitation of fluid secretion in enterospheres corresponding to conditions from A. Data \pm SEM was calculated from $n=14-40$ organoids per condition. This experiment was repeated in a total of 5 mice. Lower left panel depicts fluid secretion in WT/WT Cftr enterospheres and quantitated and represented as a dot plot graph. P-value by ANOVA with Bonferroni's multiple comparisons test. (C) Confocal images of F508del/F508del Cftr intestinal spheres treated and nontreated with STc (50 nM, 24 h) showing CFTR-specific immunostaining (green) and fluorescent labeling corresponding to F-actin (red) and nuclear counterstain (blue). (D) Representative images of R117H/R117H Cftr enterospheres depict secretion in response to forskolin stimulation under various treatment conditions: STc (50 nM, 24 h), VX-809 (2 $\mu \mathrm{M}, 24$ h), VX-770 (2 $\mu \mathrm{M}, 24$ h), STc + VX-809, and STc + VX-770. (E) Bar graph depicts fluid secretion calculated from the above-described treatment conditions. All data are represented by mean \pm SEM, calculated from $n=25-32$ organoids per condition repeated in 2 mice $P$ value by ANOVA with Bonferroni's multiple comparisons test.

CFTR allele (i.e., multiple mutations within the same CFTR gene; patient 3) (Table 1 and Figure 5, A, $\mathrm{B}$, and $\mathrm{E}-\mathrm{H})$. Patient 1 enteroids demonstrated rescue of CFTR-dependent fluid secretion upon treatment with STc (500 nM, 24 hours), VX-809, and TR (Figure 5, A and B). F508del CFTR-dependent short-circuits did not improve in primary human bronchial epithelial cells treated with STc $(500 \mathrm{nM}$, 24 hours), demonstrating the intestine-specific effect of STc on F508del CFTR correction between the airways and the gut (Figure 5, C and D). 
A

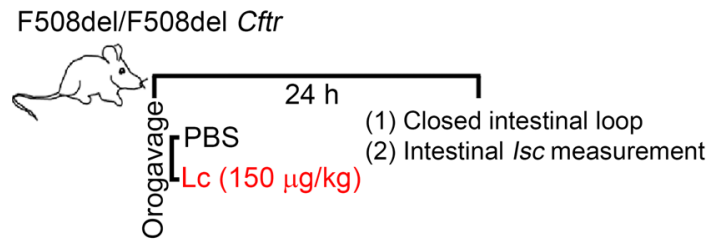

B Closed intestinal loop

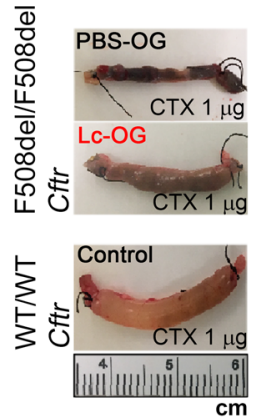

C

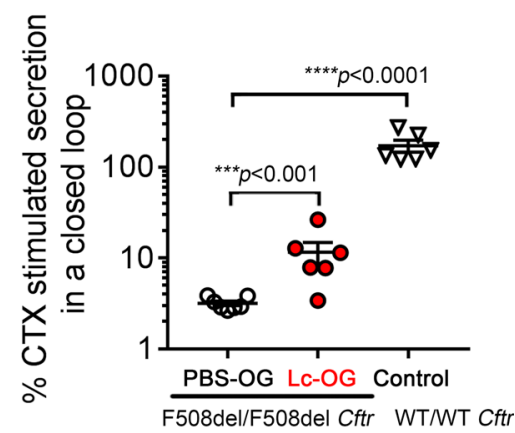

D

\section{$\underline{\text { Intestinal Isc measurement }}$ \\ F508del/F508del Cftr}
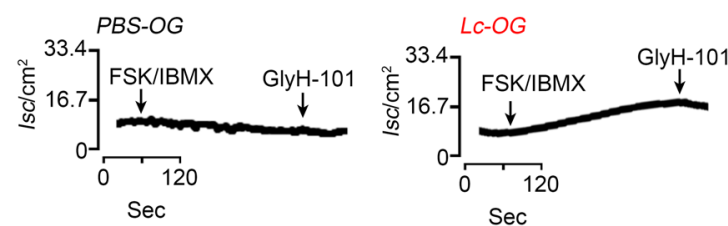

E

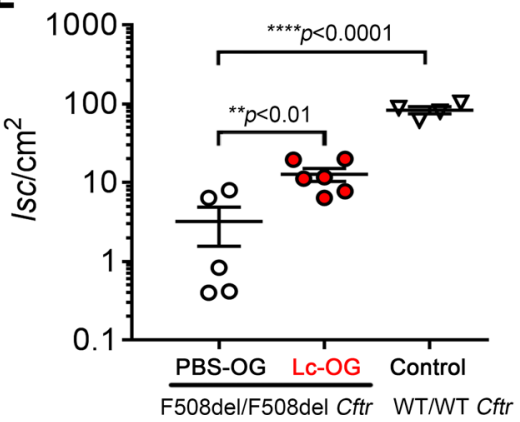

Figure 4. Linaclotide alleviates CFTR functional deficit in CF mice. (A) Experimental outline for testing the effect of orally administered LC in F508del/ F508del Cftr mice in vivo by using closed intestinal loop experiment and ex vivo by monitoring CFTR-mediated $\mathrm{Cl}^{-}$currents in isolated intestinal segments. Isc, short-circuit currents. (B) Representative images of distal intestinal loops from the small intestine of F508del/F508del Cftr mice subjected to either a PBS-OC or Lc-OG 24 hours prior to surgery with WT/WT Cftr mice as controls and injected with cholera toxin (CTX, $1 \mu \mathrm{g}$ ) to stimulate CFTR function. (C) Dot plot represents measurement of the net fluid secretion (log transformed) in the intestinal loops in response to CTX corresponding to PBS-OC ( $n=7$ ), Lc-OC $(n=6)$, and WT control $(n=6)$. All data are represented by mean \pm SEM. P value by ANOVA with Dunnett's multiple comparisons test. (D) Representative traces of murine intestinal segments isolated from F508del/F508del Cftr mice with either PBS-OC or Lc-OC. CFTR function was activated using forskolin $(10 \mu \mathrm{M})$ and IBMX $(100 \mu \mathrm{M})$ and inhibited using ClyH-101 $(100 \mu \mathrm{M})$ at the end of the experiment. (E) Dot plot represents measurement of maximal Isc increase (log transformed) in the intestinal segments corresponding to PBS-OC $(n=6)$, Lc-OC $(n=6)$, and WT control $(n=4)$. All data are represented by mean \pm SEM. $P$ value by ANOVA with Dunnett's multiple comparisons test.

While STc rescued CFTR function in patient 2 spheres, no such correctional effects were observed upon treatment with Orkambi (VX-809 + VX-770) or Kalydeco (VX-770) monotherapy (Figure 5, E and F). Functional synergism was observed between STc and VX-770 (13\% vs. $7 \%$ in the control group), suggesting that patient 2 might derive benefit from combination therapy with Kalydeco and a GCC agonist. The R117H Cftr, although retaining partial CFTR function, can be affected by poly-T/TG tract mutation such as in patient 2's case and may cause up to a $90 \%$ loss of CFTR function, as with the $5 \mathrm{~T}$ variant $(27,28)$. Importantly, a severe CF scenario has been reported to be associated with the R117H (7T/9T) variant in CFTR (29). Noteworthy is the high sweat measure ( 105 mmoles/1) of patient 2, suggesting a possibility of severe CFTR defect in this patient (Table 1 and Figure 5, E and F). Patient 3-derived intestinal spheres exhibited the highest response to Kalydeco (35\%), although CFTR function trended to increase in the presence of STc (Figure 5, G and H).

\section{Discussion}

In this study, we elucidated that administrating GCC-activating ligands rescues mutant forms of CFTR that may potentially restore intestinal fluid transport in CF patients with constipation resulting from mutant CFTR functional deficit. About $15 \%-20 \%$ of $\mathrm{CF}$ patients show signs of severe intestinal obstruction (i.e., MI, which can be treated surgically) (30). Constipation is quite common but less addressed and poorly managed in CF patients. CF GI symptoms are usually not fatal but can become a prolonged issue and need to be addressed effectively.

The available CF models, including mice and pigs, have a high degree of MI penetrance $(60 \%$ in mice and $100 \%$ in pigs), unlike humans. Murine and porcine intestines are functionally able in terms of GCC signaling but extremely poor in terms of mutant CFTR expression (31-33). According to the study by Stoltz 
Table 1. Clinical summary of 3 CF patients studied for the effects of CF modulators and STc in intestinal spheres

\begin{tabular}{|c|c|c|c|c|c|c|c|}
\hline & CFTR mutation & $\begin{array}{c}\mathrm{BMI}^{\mathrm{A}} \\
\text { percentile }\end{array}$ & $\begin{array}{c}\text { Sweat } \\
\text { (mmoles/l) }\end{array}$ & Culture & FEV1 \% & CT impression & $\mathrm{GI}$ and pancreatic phenotype \\
\hline $\begin{array}{l}\text { Patient } 1 \\
\text { (Male, } 7 \text { yrs) }\end{array}$ & F508del/F508del & $87-57$ & ${ }^{\mathrm{B} N A}$ & $\begin{array}{l}\text { MRSA, } P \text {. } \\
\text { aeruginosa } \\
\text { and } H . \\
\text { influenza }\end{array}$ & 82.6 & $\begin{array}{c}\text { Isolated subsegmental } \\
\text { bronchial wall thickening } \\
\text { and isolated subsegmental } \\
\text { atelectasis }\end{array}$ & $\begin{array}{l}\text { MI at birth with necrotizing } \\
\text { enterocolitis; pancreatic } \\
\text { insufficient; }{ }^{C} \mathrm{FE}<6\end{array}$ \\
\hline $\begin{array}{l}\text { Patient } 2 \\
\text { (Male, } 15 \text { yrs) }\end{array}$ & $\begin{array}{l}\text { F508del/R117H } \\
\text { (7T/9T) }\end{array}$ & $65-95$ & 105 & None & 112 & $\begin{array}{l}\text { No bronchiectasis, mild } \\
\text { peribronchial thickening, } \\
\text { incidental small, interior } \\
\text { mediastinal nodes, } \\
\text { possibly reactive }\end{array}$ & $\begin{array}{c}\text { Recurrent pancreatitis; }{ }^{\mathrm{C} F E} \\
\text { intermediate = } 232 \mu \mathrm{g} / \mathrm{g} \text {; normal } \\
\text { trypsin, amylase, lipase and } \\
\text { chymotrypsin }\end{array}$ \\
\hline $\begin{array}{l}\text { Patient } 3 \\
\text { (Male, } 10.3 \\
\text { yrs) }\end{array}$ & $\begin{array}{l}\text { G542X/R74W } \\
\text { V201M D1270N }\end{array}$ & $45-58$ & 72 & None & 110 & $\begin{array}{l}\text { Previously identified mild } \\
\text { multifocal bronchiectasis } \\
\text { has essentially resolved }\end{array}$ & $\begin{array}{l}\text { History of constipation; } \\
\text { pancreatic sufficient; ' } F E \text { > } 500\end{array}$ \\
\hline
\end{tabular}

A2016-2017. B Not available. ${ }^{C}$ Fecal elastase $\mu \mathrm{g} / \mathrm{g}$ stool. FEV1, forced expiratory volume in 1 second.

and colleagues, at least $20 \%$ of WT CFTR transcripts, which will produce normal functional CFTR, and > 25\% of WT CFTR-mediated anion secretion are needed to prevent MI in F508del/F508del Cftr pigs (32). The protein expression of F508del CFTR in mice and pigs ( $7 \%$ of WT CFTR) falls well below the $20 \%$ threshold. Furthermore, the physiological stimulation of F508del CFTR is expected to be much more diminished compared with WT CFTR, suggesting that MI is expected to be highly penetrant in these CF species (33). Consistently, the GI manifestations in Cftr-KO pigs are strikingly similar to those in Cftr ${ }^{\text {F508del/F508del }}$ pigs. The presence of modifier genes and environmental influence may be a genetic advantage in humans for $\mathrm{MI}$ over genetically unmixed CF mice and pigs raised in a controlled environment (34). According to a previously published study, higher scores of MI prevalence associate with more severe CF mutations (G542X [0.31], F508del [0.22], and G551D [0.08]) and clinical phenotypes (pancreatic insufficiency and low forced expiratory volumes [FEVs]) (30). Hence, there is an evident genotype-phenotype correlation between CFTR expression and MI. Prior studies suggest that intestinal F508del CFTR can be partially processed to form band $\mathrm{C}$ in humans and pigs $(33,35,36)$. Whether GCC signaling in the gut renders the potential of intestinal epithelial cells in these species to process mutant CFTR protein warrants accumulating more experimental evidences.

The intestinal stem culture system to generate organoids has become a valuable tool to study structural and functional aspects of the intestine ex vivo and can potentially be modeled to study genetic and infectious human diseases $(37,38)$. This intestinal culture model and its capability as a robust assay of CFTR functional assessment allow estimating the CF modulator response ex vivo and develop personalized medicine for CF patients. In this study, we demonstrated the feasibility of testing the potential CF therapeutic agent Lc in the enterospheres derived from CF subjects with different CFTR mutations and pathological manifestations. Importantly, all 3 tested subject enterospheres responded positively to STc/Lc. Hence, the type of CF mutation does not become a limiting factor in therapeutic application of STc/Lc.

\section{Methods}

Mice

F508del/F508del Cftr mice used in the study were either from in-house colonies or provided by Craig Hodges (University of North Carolina, Chapel Hill, North Carolina, USA). The strain was originally generated by Kirk R. Thomas at the University of Utah (Salt Lake City, Utah, USA) (39). The mice were maintained in a barrier facility at CCHMC and were fed normal chow, but they were treated with water containing an osmotic laxative (Colyte) composed of Polyethylene glycol 3350 (18 mM), NaCl (25 mM), $\mathrm{KCl}(10 \mathrm{mM}), \mathrm{NaHCO}_{3}(20 \mathrm{mM})$ and anhydrous $\mathrm{Na}_{2} \mathrm{SO}_{4}(40 \mathrm{mM})$.

Intestinal fluid secretion (in vivo) measurement or closed intestinal loop experiment F508del/F508del Cftr mice (body weight 20-22 g) were oral gavaged with either PBS or Lc (150 $\mu \mathrm{g} /$ $\mathrm{kg}$ ) and were deprived of food for 24 hours prior to surgery. Mice were anesthetized under isofluorane, 
A Patient 1- F508del/F508del CFTR

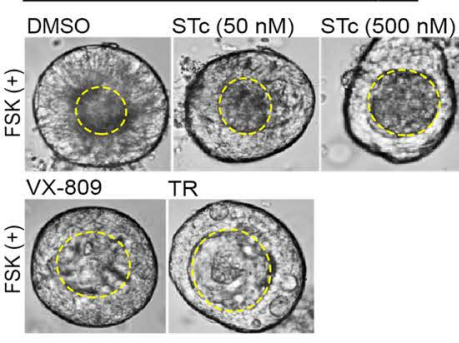

C

Primary human bronchial epithelial cells (ISC)

F508del/F508del cftr

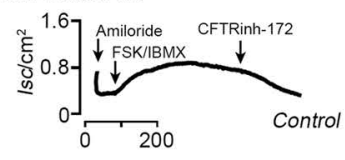

$\mathrm{Sec}$

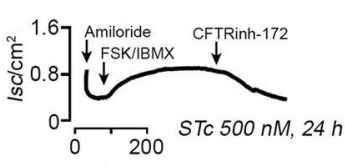

$\mathrm{Sec}$
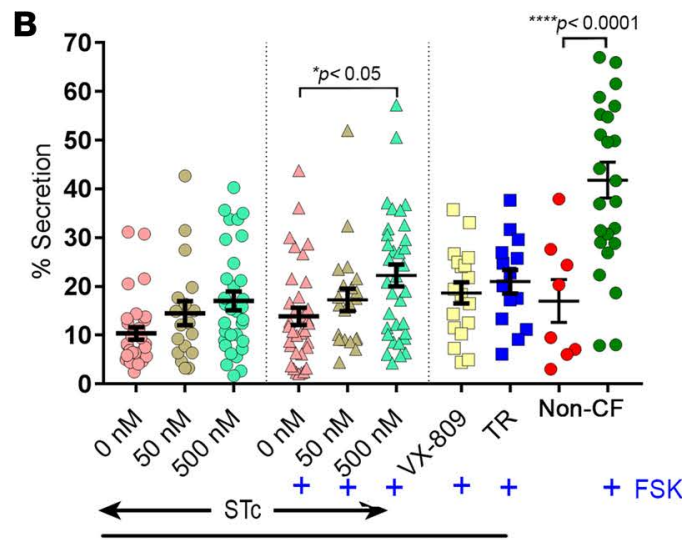

F508del/F508del CFTR
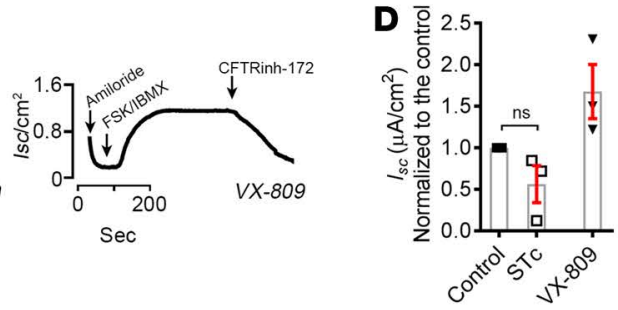

E Patient 2- F508del/R117H(7T/9T)CFTR
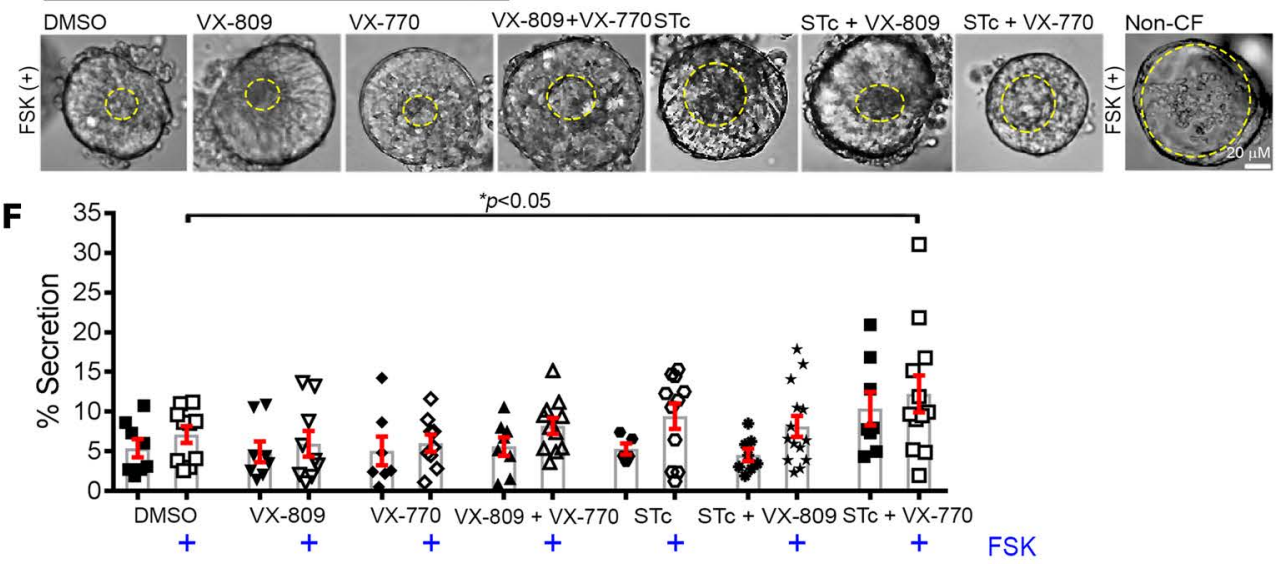

G Patient 3- G542X/R74W, V201M, D1270NCFTR H
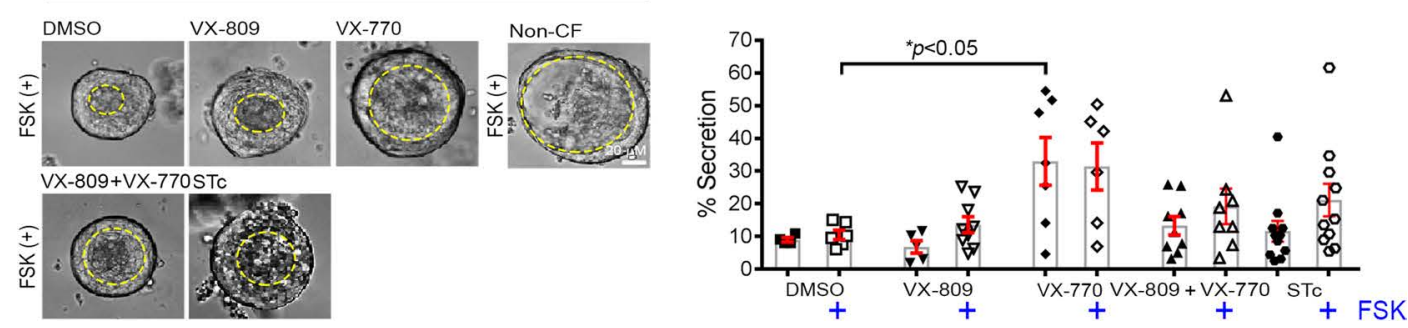

Figure 5. GCC agonism improves fluid secretion in CF patient-derived enterospheres. (A) Representative images of CF patient 1-derived F508del/ F508del CFTR enterospheres depict secretion in response to forskolin stimulation under various treatment conditions: STc (50 nM and $500 \mathrm{nM}$, 24 h), VX-809 ( $2 \mu \mathrm{M}, 24 \mathrm{~h})$, and temperature rescue (TR). (B) Dot plot depicts fluid secretion calculated from $n=15-32$ enterospheres corresponding to the treatment conditions described in $\mathbf{A}$. Data represents mean \pm SEM with $P$ value calculated using 1-way ANOVA with Bonferroni's multiple comparisons test. (C) Representative traces of short-circuit current (Isc) (left) in primary human bronchial epithelial cells carrying F508del/F508del CFTR untreated or treated with STc $(500 \mathrm{nM}, 24 \mathrm{~h})$ or VX-809 $(2.5 \mu \mathrm{M}, 24 \mathrm{~h})$. CFTR-dependent chloride transport was stimulated using forskolin (10 $\mu \mathrm{M})$ and IBMX $(100 \mu \mathrm{M})$ and inhibited with CFTRinh-172 added at the end of the experiment. (D) Bar graph represents quantitation of Isc measured in above-described treatment conditions ( $n=3$ independent experiments) and normalized to the untreated control. Data represents mean \pm SEM. $P$ value calculated by 1-way ANOVA with Bonferroni's adjustment. (E) Representative images of CF patient 2-derived F508del/R117H 7T/9T CFTR enterospheres depict secretion in response to forskolin stimulation under various treatment conditions: VX-809 ( $2 \mu \mathrm{M}, 24 \mathrm{~h}), \mathrm{VX}-770$ ( $2 \mu \mathrm{M}, 24 \mathrm{~h})$, VX-770 + VX-809, STc (50 nM, 24 h), STc + VX-809, and STc + VX-770. (F) Bar graph depicts fluid secretion calculated from $n=5-14$ enterospheres corresponding to the treatment conditions described in $\mathbf{E}$. Data represents mean \pm SEM with $P$ value calculated using 1-way ANOVA with Bonferroni's multiple comparisons test. (G) Representative images of CF patient 3-derived G542X/ R74W, V201M, D1270N CFTR enterospheres depict 
secretion in response to forskolin stimulation under various treatment conditions: VX-809 (2 $\mu \mathrm{M}, 24 \mathrm{~h}), \mathrm{VX}-770(2 \mu \mathrm{M}, 24 \mathrm{~h}), \mathrm{VX}-770+\mathrm{VX}-809$, and STc (50 nM, 24 h). (H) Bar graph depicts fluid secretion calculated from $n=6-9$ enterospheres corresponding to the conditions described in G. Yellow circles indicate luminal area. Data represents mean \pm SEM with $P$ value calculated using 1-way ANOVA with Bonferroni's multiple comparisons.

and physiological conditions were maintained $\left(\mathrm{O}_{2}\right.$ and body temperature $)$ throughout surgery. A small abdominal incision was made to expose the distal region of small intestine. Intestinal loops $(\sim 2 \mathrm{~cm})$ were exteriorized and isolated (2 loops per mouse). The closed loops were then injected with $100 \mu$ of PBS alone or PBS containing CTX $(1 \mu \mathrm{g})$. The abdominal incision and skin incision were closed with surgical sutures, and the mice were allowed to recover. After 6 hours, the mice were sacrificed by $\mathrm{CO}_{2}$. Intestinal loops were removed, and loop fluid weight was measured to quantitate net fluid secretion.

\section{Chemicals and antibodies}

Chemicals used in this study include Forskolin, 8-Br cGMP, IBMX, GlyH-101, amiloride, and CTX from MilliporeSigma and CFTRinh-172 from Tocris, VX-809, and VX-770 from Selleckchem.

Antibodies in this study included anti-CFTR antibodies R1104 (Eric Sorscher lab, CF Center, University of Alabama, Birmingham, Alabama, USA [presently, Emory University, Atlanta, Georgia, USA]), 24-1 (MAB25031, R\&D Systems), and CF3 (ab2784, Abcam); anti-HA (3724, Cell Signaling Technologies); and anti-FLAG HRP (A8592, clone M2, MilliporeSigma). STc was custom synthesized from American Peptide Company. Chloride indicator SPQ was purchased from (Thermo Fisher Scientific).

\section{Cell transfection}

HEK 293 cells were obtained from ATCC and were prepared in regular medium (DMEM/F-12 10\% FBS, $1 \%$ penicillin-streptomycin). Human GCC cDNA was a gift from Kris A. Steinbrecher (CCHMC). Cells were transfected with human HA-GCC (HA tag was inserted at amino-acid position 39) cDNA by using Lipofectamine 3000 (Thermo Fisher Scientific) according to the manufacturer's protocol. Transfected cells were studied after 48 hours.

\section{Cell lysate preparation and CFTR immunoprecipitation}

HEK 293 cells stably expressing FLAG-F508del CFTR were lysed in PBS 0.2\% Triton X-100 containing protease-inhibitor cocktail ( $1 \mu \mathrm{M}$ aprotonin, $1 \mu \mathrm{M}$ leupeptin, and $1 \mathrm{mM}$ phenylmethylsulfonyl fluoride). FLAG-CFTR was immunoprecipitated from whole-cell lysates in 2 ways: first, by using FLAG-conjugated resin (MilliporeSigma) and, second, by cross-linking 24-1 CFTR antibody to protein A agarose ( $0.5 \mu \mathrm{g}$ antibody per $10 \mu \mathrm{l}$ of protein $\mathrm{A}$-conjugated resin by using disuccinimidyl suberate cross-linker). Proteins immobilized on beads were eluted using a low-pH, glycine-based elution buffer containing $0.2 \%$ Triton-X-100. Samples were incubated for 10 minutes at $37^{\circ} \mathrm{C}$ and subjected to SDS-PAGE and Western blot following standard protocols.

\section{Intestinal crypt isolation and quantitation of fluid secretion in enterospheres}

Preparation of mouse intestinal crypt and quantitation have been thoroughly described previously (25). Human crypts were isolated and cultured as reported earlier (40). For fluid secretion measurement with the high-content microscope, total organoid area was calculated before and after forskolin treatment.

\section{Immunofluorescence}

Mouse intestinal organoids were fixed using 3.7\% formaldehyde for 10 minutes at $25^{\circ} \mathrm{C}$. Cells were then washed 2 times with $1 \times$ PBS and embedded in warm HistoGel (Thermo Fisher Scientific). Cells were permeabilized using PBS $0.3 \%$ Triton X-100 for 30 minutes at $25^{\circ} \mathrm{C}$. After a brief wash, cells were blocked with goat serum (Biocare Medical) for 1 hour at $25^{\circ} \mathrm{C}$. Intestinal organoids were incubated with CF3 antibody (1:25) or GCC antibody (1:50) (41) at $4^{\circ} \mathrm{C}$ overnight, washed with PBS $0.05 \%$ Tween buffer, and incubated with mouse secondary antibody (Alexa Fluor 488 or 568, 1:500, Thermo Fisher Scientific) for 1 hour at $25^{\circ} \mathrm{C}$. For F-actin staining, phalloidin 488 was used (Thermo Fisher Scientific), and - in the final step — intestinal structures were mounted in Vectashield mounting medium with DAPI (Vector Labs). Stained intestinal organoids were examined using a confocal microscope (Olympus FV1200). 
SPQ assay

HEK 293 cells $\left(1 \times 10^{6}\right)$ expressing no CFTR, WT CFTR, and F508del CFTR \pm GCC treated with STc $(500 \mathrm{nM}$, 24 hours) were plated in a clear bottom black well plate. Cells were treated with $10 \mathrm{mM}$ SPQ prepared in 1:1 OptiPrep medium/water solution for 10 minutes at $37^{\circ} \mathrm{C}$ (Thermo Fisher Scientific). Cells were washed once with 130 $\mathrm{mM}$ NaI prepared in SPQ buffer (20 mM HEPES, $10 \mathrm{mM}$ glucose, $4 \mathrm{mM} \mathrm{KNO}_{3}, 1 \mathrm{mM} \mathrm{Ca}\left[\mathrm{NO}_{3}\right]_{2} \cdot 4 \mathrm{H}_{2} \mathrm{O}$, and 1 $\mathrm{mM} \mathrm{Mg}\left[\mathrm{NO}_{3}\right]_{2} \cdot 6 \mathrm{H}_{2} \mathrm{O}$ ). Cells were incubated in NaI buffer for 20 minutes each for a total of 3 times at $37^{\circ} \mathrm{C}$. Cells were washed once in $130 \mathrm{mM} \mathrm{NaNO}_{3}$ prepared in SPQ buffer. For baseline measurement, cells were incubated with $130 \mathrm{mM} \mathrm{NaNO}_{3}$ buffer, and fluorescence was measured at $360_{\mathrm{Ex}} / 460_{\mathrm{Em}}$ for a total of 5 time points in Flexstation 3 (Molecular Devices). CFTR-activating agent forskolin $(10 \mu \mathrm{M})$ was added to the cells, and readings were taken for a total of 10 time points. Signal was quenched using $130 \mathrm{mM} \mathrm{NaI}$ buffer at the end of the experiment.

\section{Short-circuit current measurements}

Polarized human bronchial epithelial cells cultured on Costar Transwell permeable supports (filter diameter $12 \mathrm{~mm}$ ) were mounted in an Ussing chamber system (Physiologic Instruments) maintained at $37^{\circ} \mathrm{C}(42)$. Epithelial cells were bathed in Ringer's solution (mM) (basolateral side: $140 \mathrm{NaCl}, 5 \mathrm{KCl}, 0.36 \mathrm{~K}_{2} \mathrm{HPO}$,

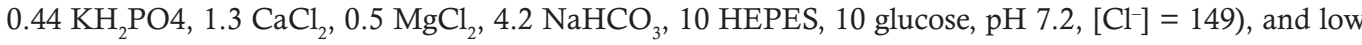
$\mathrm{Cl}^{-}$Ringer's solution (mM) (apical side: $133.3 \mathrm{Na}$-gluconate, $2.5 \mathrm{NaCl}, 0.36 \mathrm{~K}_{2} \mathrm{HPO}$, $0.44 \mathrm{KH}_{2} \mathrm{PO} 4,5.7$ $\mathrm{CaCl}_{2}, 0.5 \mathrm{MgCl}_{2}, 4.2 \mathrm{NaHCO}_{3}, 10 \mathrm{HEPES}, 10$ mannitol, $\left.\mathrm{pH} 7.2,\left[\mathrm{Cl}^{-}\right]=14.8\right)$ in the presence or absence of VX-809 $(2.5 \mu \mathrm{M})$ and STc $(500 \mathrm{nM})$ at $37^{\circ} \mathrm{C}$ and saturated with $95 \% \mathrm{O}_{2}$ and $5 \% \mathrm{CO}_{2}$. Cells were treated first with amiloride $(50 \mu \mathrm{M})$, and after current stabilization, CFTR was activated by adding $10 \mu \mathrm{M}$ forskolin and $100 \mu \mathrm{M}$ IBMX on the apical side. CFTRinh-172 $(20-50 \mu \mathrm{M})$ was added to the apical side at the end of the experiment to verify CFTR dependency of the currents.

Distal intestinal segments were isolated from F508del/F508del Cftr mice oral gavaged 24 hours earlier with either PBS or Lc $(150 \mu \mathrm{g} / \mathrm{kg})$ and WT/WT Cftr mice as controls and were bathed on both surfaces in the Ussing setup with a solution containing (in $\mathrm{mM}$ ) $140 \mathrm{NaCl}, 5 \mathrm{KCl}, 0.36 \mathrm{~K}_{2} \mathrm{HPO} 4,0.44 \mathrm{KH}_{2} \mathrm{PO} 4,1.3$ $\mathrm{CaCl}_{2}, 0.5 \mathrm{MgCl}_{2}, 4.2 \mathrm{NaHCO}_{3}, 10 \mathrm{HEPES}, \mathrm{pH} 7.2$, at $37^{\circ} \mathrm{C}$ and gassed with $5 \% \mathrm{CO}_{2}$. CFTR was activated using apical $10 \mu \mathrm{M}$ forskolin and $100 \mu \mathrm{M}$ IBMX and inhibited with apical $100 \mu \mathrm{M}$ GlyH-101.

\section{Measurement of whole-cell cGMP}

HEK 293 cells expressing HA-GCC or without an expression of GCC were treated with STc $500 \mathrm{nM}$ and IBMX for 30 minutes at $37^{\circ} \mathrm{C}$. F508del/F508del Cftr and WT/WT Cftr murine enterospheres were treated with STc and Lc (500 nM each) for 60 minutes at $37^{\circ} \mathrm{C}$ in the presence of IBMX. Cells were lysed in $0.1 \mathrm{~N} \mathrm{HCl} 0.2 \%$ Triton-X-100 and centrifuged at $800 \mathrm{~g}$, and the supernatant was collected and used for cGMP-specific ELISA following the manufacturer's protocol (Enzo Life Sciences).

\section{Biotin-Lc tracking}

For Q dot-based biotin-Lc tracking, HEK 293 cells with and without stable expression of HA-GCC were incubated with biotin-Lc $(2 \mu \mathrm{M})$ for 30 minutes in no phenol red DMEM medium at $37^{\circ} \mathrm{C}$ and washed 3 times with the same medium. Streptavidin-conjugated Q dot 655 (1:1,000 dilution, Thermo Fisher Scientific) was added to the medium and incubated for 5 minutes. Cells were subsequently washed 6 times with no phenol red DMEM medium and imaged on an inverted Olympus wide-field microscope (IX51) in a temperature-controlled environment $\left(37^{\circ} \mathrm{C}\right)$.

For biotin-Lc tracking, intestinal organoids were incubated with biotin-Lc $(2 \mu \mathrm{M})$ for the time points 1 minute, 5 minutes, and 60 minutes at $37^{\circ} \mathrm{C}$ in HBSS and, at the end of the time point, immediately washed 3 times with HBSS and fixed in $4 \%$ formaldehyde. A set of organoids was kept at $4^{\circ} \mathrm{C}$ and incubated for 60 minutes with biotin-Lc $(2 \mu \mathrm{M})$. Organoids were permeabilized using $0.3 \%$ Triton-X-100 for 30 minutes and blocked in 2\% BSA PBS-Tween at room temperature. Organoids were incubated with streptavidin-conjugated Texas red (1:250) and incubated for 2 hours at room temperature. Organoids were washed 3 times with PBSTween, mounted in DAPI-containing medium, and imaged using a confocal microscope (Olympus FV1200).

\section{Real time PCR}

RNA was extracted using Qiagen RNeasy Mini Kit. cDNA was synthesized using SuperScript III Reverse Transcriptase (Thermo Fisher Scientific). Sybr green-based real time PCR was performed using prevalidated primers for different murine genes (mCFTR, mNHE3, mLgr5, mHistone H3, and mGAPDH). 


\section{Statistics}

The statistical significance was calculated using 1-way ANOVA with appropriate adjustment of $P$ value in experiments with multiple groups. $P<0.05$ was considered as statistically significant. All the experiments were repeated in at least triplicates.

\section{Study approval}

Human studies. Patient studies for research were approved by CCHMC IRB under 2011-2616. A written consent was obtained from all the participants.

Mice studies. All procedures in mice were performed in compliance with institutional guidelines and were approved by CCHMC's IACUC.

\section{Author contributions}

KA conceived, designed, and performed the experiments and wrote the manuscript. YH and KM assisted in the microinjection experiments and SPQ assay. SY designed the recombinant cDNA constructs. NS and $\mathrm{MH}$ prepared the human organoids from $\mathrm{CF}$ patients. MMK, GH, CBK, and ISS provided study reagents and assisted in the study design. KAS provided the GCC-KO mice, human GCC cDNA, and critical insights into the research design. JPC provided patient samples and the clinical data summary. APN conceived and supervised the project, designed the experiments, and edited the draft manuscript. JJP obtained duodenal biopsies from CF patients.

\section{Acknowledgments}

Authors are grateful to David Armbruster for editing the manuscript. This research was funded by the NIH (to APN; DK080834 and DK093045), CF Foundation grants to KA (ARORA16F0) and APN (NAREN 14XX0 and CLANCY15R0), and Ironwood Pharmaceuticals.

Address correspondence to: Anjaparavanda P. Naren, 3333 Burnet Avenue MLC 2021, Cincinnati Children's Hospital Medical Center, Cincinnati, Ohio 45229, USA. Phone: 513.803.4731; Email: anaren@cchmc.org.

1. Dalemans W, et al. Altered chloride ion channel kinetics associated with the delta F508 cystic fibrosis mutation. Nature. 1991;354(6354):526-528.

2. Drumm ML, et al. Chloride conductance expressed by delta F508 and other mutant CFTRs in Xenopus oocytes. Science. 1991;254(5039):1797-1799.

3. Sabharwal S. Gastrointestinal Manifestations of Cystic Fibrosis. Gastroenterol Hepatol (N Y). 2016;12(1):43-47.

4. Olivier AK, Gibson-Corley KN, Meyerholz DK. Animal models of gastrointestinal and liver diseases. Animal models of cystic fibrosis: gastrointestinal, pancreatic, and hepatobiliary disease and pathophysiology. Am J Physiol Gastrointest Liver Physiol. 2015;308(6):G459-G471.

5. Basu N, Arshad N, Visweswariah SS. Receptor guanylyl cyclase C (GC-C): regulation and signal transduction. Mol Cell Biochem 2010;334(1-2):67-80.

6. Schulz S, Green CK, Yuen PS, Garbers DL. Guanylyl cyclase is a heat-stable enterotoxin receptor. Cell. 1990;63(5):941-948

7. Gabriel SE, Brigman KN, Koller BH, Boucher RC, Stutts MJ. Cystic fibrosis heterozygote resistance to cholera toxin in the cystic fibrosis mouse model. Science. 1994;266(5182):107-109.

8. Field M. Intestinal ion transport and the pathophysiology of diarrhea. J Clin Invest. 2003;111(7):931-943.

9. Navaneethan U, Giannella RA. Mechanisms of infectious diarrhea. Nat Clin Pract Gastroenterol Hepatol. 2008;5(11):637-647.

10. Fiskerstrand T, et al. Familial diarrhea syndrome caused by an activating GUCY2C mutation. N Engl J Med. 2012;366(17):1586-1595

11. Romi H, et al. Meconium ileus caused by mutations in GUCY2C, encoding the CFTR-activating guanylate cyclase $2 \mathrm{C}$. Am $J$ Hum Genet. 2012;90(5):893-899.

12. Chey WD, et al. Linaclotide for irritable bowel syndrome with constipation: a 26-week, randomized, double-blind, placebo-controlled trial to evaluate efficacy and safety. Am J Gastroenterol. 2012;107(11):1702-1712.

13. Johnston JM, Shiff SJ, Quigley EM. A review of the clinical efficacy of linaclotide in irritable bowel syndrome with constipation. Curr Med Res Opin. 2013;29(2):149-160.

14. Jensen TJ, Loo MA, Pind S, Williams DB, Goldberg AL, Riordan JR. Multiple proteolytic systems, including the proteasome, contribute to CFTR processing. Cell. 1995;83(1):129-135.

15. Van Goor F, et al. Correction of the F508del-CFTR protein processing defect in vitro by the investigational drug VX-809. Proc Natl Acad Sci U S A. 2011;108(46):18843-18848.

16. Okiyoneda T, et al. Mechanism-based corrector combination restores $\triangle$ F508-CFTR folding and function. Nat Chem Biol. 2013;9(7):444-454.

17. Ramsey BW, et al. A CFTR potentiator in patients with cystic fibrosis and the G551D mutation. NEngl J Med. 2011;365(18):1663-1672.

18. Ronan NJ, Fleming C, O'Callaghan G, Maher MM, Murphy DM, Plant BJ. The role of ivacaftor in severe cystic fibrosis in a 
patient with the R117H mutation. Chest. 2015;148(3):e72-e75.

19. Gentzsch M, et al. Restoration of R117H CFTR folding and function in human airway cells through combination treatment with VX-809 and VX-770. Am J Physiol Lung Cell Mol Physiol. 2016;311(3):L550-L559.

20. Sato T, et al. Single Lgr5 stem cells build crypt-villus structures in vitro without a mesenchymal niche. Nature. 2009;459(7244):262-265.

21. Sato T, Clevers H. Growing self-organizing mini-guts from a single intestinal stem cell: mechanism and applications. Science. 2013;340(6137):1190-1194.

22. Foulke-Abel J, et al. Human enteroids as a model of upper small intestinal ion transport physiology and pathophysiology. Gastroenterology. 2016;150(3):638-649.e8.

23. Dekkers JF, et al. A functional CFTR assay using primary cystic fibrosis intestinal organoids. Nat Med. 2013;19(7):939-945.

24. Arora K, et al. Stabilizing rescued surface-localized $\delta$ f508 CFTR by potentiation of its interaction with $\mathrm{Na}(+) / \mathrm{H}(+)$ exchanger regulatory factor 1. Biochemistry. 2014;53(25):4169-4179.

25. Moon C, et al. Compartmentalized accumulation of cAMP near complexes of multidrug resistance protein 4 (MRP4) and cystic fibrosis transmembrane conductance regulator (CFTR) contributes to drug-induced diarrhea. J Biol Chem. 2015;290(18):11246-11257.

26. Denning GM, Anderson MP, Amara JF, Marshall J, Smith AE, Welsh MJ. Processing of mutant cystic fibrosis transmembrane conductance regulator is temperature-sensitive. Nature. 1992;358(6389):761-764.

27. de Nooijer RA, et al. Assessment of CFTR function in homozygous R117H-7T subjects. J Cyst Fibros. 2011;10(5):326-332.

28. Kiesewetter S, et al. A mutation in CFTR produces different phenotypes depending on chromosomal background. Nat Genet. 1993;5(3):274-278.

29. Thauvin-Robinet C, et al. Severe cystic fibrosis with R117H/DF508 and 7T/9T polymorphism: difficulties in genetic counselling after newborn screening. Presented at: 2006 ASHG Annual Meeting; October 9-13, 2006; New Orleans, Lousiana, USA. Program 671.

30. Dupuis A, et al. Prevalence of meconium ileus marks the severity of mutations of the Cystic Fibrosis Transmembrane Conductance Regulator (CFTR) gene. Genet Med. 2016;18(4):333-340.

31. Bijvelds MJ, et al. Inhibition of Heat-Stable Toxin-Induced Intestinal Salt and Water Secretion by a Novel Class of Guanylyl Cyclase C Inhibitors. J Infect Dis. 2015;212(11):1806-1815.

32. Stoltz DA, et al. Intestinal CFTR expression alleviates meconium ileus in cystic fibrosis pigs. J Clin Invest. 2013;123(6):2685-2693

33. Ostedgaard LS, et al. The $\Delta$ F508 mutation causes CFTR misprocessing and cystic fibrosis-like disease in pigs. Sci Transl Med. 2011;3(74):74ra24

34. Blackman SM, et al. Relative contribution of genetic and nongenetic modifiers to intestinal obstruction in cystic fibrosis. Gastroenterology. 2006;131(4):1030-1039.

35. Kälin N, Claass A, Sommer M, Puchelle E, Tümmler B. DeltaF508 CFTR protein expression in tissues from patients with cystic fibrosis. J Clin Invest. 1999;103(10):1379s-1389.

36. van Barneveld A, et al. Functional analysis of F508del CFTR in native human colon. Biochim Biophys Acta. 2010;1802(11):1062-1069.

37. Clevers H. Modeling development and disease with organoids. Cell. 2016;165(7):1586-1597.

38. Riemer P, et al. Oncogenic $\beta$-catenin and PIK3CA instruct network states and cancer phenotypes in intestinal organoids. $J$ Cell Biol. 2017;216(6):1567-1577.

39. Zeiher BG, et al. A mouse model for the delta F508 allele of cystic fibrosis. J Clin Invest. 1995;96(4):2051-2064.

40. Mahe MM, Sundaram N, Watson CL, Shroyer NF, Helmrath MA. Establishment of human epithelial enteroids and colonoids from whole tissue and biopsy. J Vis Exp. 2015;(97).

41. Mann EA, Jump ML, Wu J, Yee E, Giannella RA. Mice lacking the guanylyl cyclase C receptor are resistant to STa-induced intestinal secretion. Biochem Biophys Res Commun. 1997;239(2):463-466.

42. Li C, et al. Spatiotemporal coupling of cAMP transporter to CFTR chloride channel function in the gut epithelia. Cell. 2007;131(5):940-951. 